\title{
Inter-organizational Systems: From Business Values over Business Processes to Deployment
}

\author{
Christian Huemer ${ }^{1}$, Philipp Liegl ${ }^{1}$, Rainer Schuster ${ }^{2}$, Hannes Werthner ${ }^{2}$, and Marco Zapletal ${ }^{2}$ \\ ${ }^{1}$ Business Informatics Group, ${ }^{2}$ Electronic Commerce Group \\ Institute of Software Technology and Interactive Systems, Vienna University of Technology, Austria \\ Email: \{huemer, liegl\}@big.tuwien.ac.at, \{schuster, werthner, marco\}@ec.tuwien.ac.at
}

\begin{abstract}
Inter-organizational systems have significantly been affected by Service-oriented Architectures (SOA) and Web Services - the state-of-the-art technology to implement SOA. SOA is said to enable quick and inexpensive changes of the IT in order to establish new business partnerships or to reflect changes in existing partnerships. However, current approaches to inter-organizational systems focus too much on existing Web Services standards and, thus, on the technology layer. In such an approach the technology drives the business. In this paper we analyze the shortcomings of this bottom-up approach. As an alternative we suggest a top-down methodology where the business requirements drive the technology. This methodology starts off with the business value perspective, leading to a business process perspective and resulting in an IT execution perspective. We do not invent any new approaches on each of these layers, rather we outline how existing approaches are used and combined into a business requirements driven approach to inter-organizational systems.
\end{abstract}

Index Terms-business modeling, business process modeling, inter-organizational systems, service-oriented architectures, semantically-enriched business processes

\section{INTRODUCTION}

In order to stay in business, companies must quickly adopt to faster and faster changing business conditions. Business models must reflect these changes, business processes must be designed supporting the value exchanges, and IT applications must adjust to changing company goals. Management expects this to happen at low cost. Serviceoriented Architectures (SOA) have the potential to provide a new level of flexibility in regard to the adaptation of the affected IT systems. Whereas in former days change requests to the IT resulted in a rigorous change of IT system implementations, the more and more service-oriented IT departments nowadays focus the challenge of service reconfiguration and service alignment. Service alignment refers to the reconcilement between business partners to provide complementary services. Whereas service reconfiguration refers to the internal adjustments of a business partner in delivering a composite service.

In order to analyze the potential of SOA, we first have to understand what SOA refers to. According to the OASIS SOA Reference Model [1], SOA stands for a paradigm for organizing and utilizing distributed capabilities that may be under the control of different ownership domains. This specification continues that in general, entities (people and organizations) create capabilities to solve the problems they face in the course of their business. The main drivers for a SOA are managing the growth of large-scale enterprise sys- tems, facilitating Internet-scale provision and use of services, and reducing costs in inter-organizational cooperation.

From the above excerpts of the SOA Reference Model it becomes evident that SOA is not limited to implementation issues addressed by Web Services - the current technology of choice to implement a SOA. This means that a SOAbased approach to inter-organizational cooperation must also address the business requirements in organizing and utilizing a distributed solution for a business partnership. This is in line with the Open-edi reference model, which became an ISO standard for inter-organizational systems in the mid 1990ies [2]. Open-edi distinguishes between the business operational view (BOV) and the functional service view (FSV). The BOV addresses the business aspects such as business information, business conventions, agreements and rules among organizations. The FSV is related to information technology aspects which are necessary to support the execution of a business collaboration. Accordingly, the FSV implements the scenarios developed in the BOV.

At first sight one might tend to reduce the BOV layer to model the business interactions between the involved business partners. However, it is at least equally important to analyze the value propositions of each of the participating business partners [3]. Consequently, separating the concerns in developing inter-organizational systems based on SOA results in three different perspectives shown in figure 1 . The management focuses on the value perspective described by business models. Business people have a process perspective described by business process models that operationalize the business models. The IT folks focus on the execution perspective of the deployment artifacts implementing the business process models.

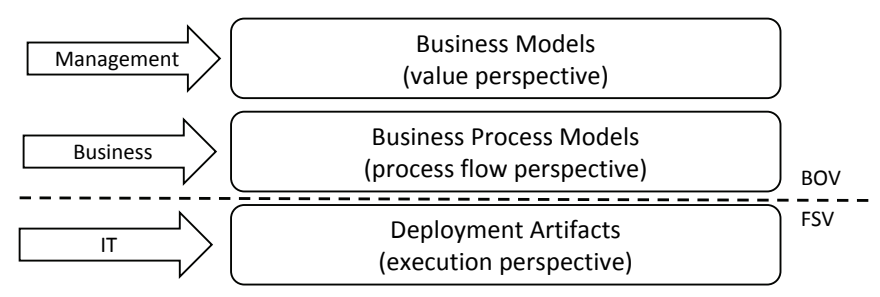

Fig. 1. Layers of the BSopt approach

These three layers are addressed by the project BSopt Business Semantics on top of process technology, which is funded under the 4th Semantic Systems Call of the Austrian Research Promotion Agency. The project aims at developing 
a methodology and a tool set for a top-down approach for SOA where the business requirements drive the underlying IT infrastructure implemented by Web Services.

The rest of the paper is organized as follows: In section II we analyze the shortcomings of existing SOA approaches. In particular, we illustrate the potential failures of a bottomup approach starting from a Web Services-centric view to develop a SOA. Section III outlines the research goals of the BSopt approach. Since the BSopt methodology will be built by an amalgation of existing approaches - each addressing a different perspective of figure 1 - we present these approaches in section IV on related work. Section V highlights the approach considered by BSopt to deliver a topdown methodology and tool support for inter-organizational systems. A short summary in section VI concludes the paper.

\section{SHORTCOMINGS OF TRADITIONAL APPROACHES}

Traditional approaches in the area of SOA have several shortcomings in supporting inter-organizational systems. In this section we elaborate the most significant limitations which we are going to tackle with the BSopt methodology.

\section{A. Complexity goes beyond simple Web Services model}

Web Services are certainly an appropriate technology to implement inter-organizational business processes. However, the traditional approach to implement Web Services may not be appropriate as we elaborate in the following: Businesses provide access to application components that implement key functionalities via the Internet. These application components are known as Web Services and their interfaces are described by Web Service Definition Language (WSDL) artifacts. A provider of a service is expected to register all the details of accessing the service. Potential service consumers may search the registry for a suitable service and retrieve its access information. By following the access information the service consumer binds its application to the service offered by the service provider. This rather simplistic approach might work for simple services that have no dependencies on other services executed between the service consumer and the service provider - but it still needs semantically enhanced service descriptions. In practice, even this relatively simple case is not working as envisioned - this is underpinned by shutting down the public UDDI directories previously offered by Microsoft, IBM, and SAP in 2005.

An inter-organizational business process is much more complicated than a simple service request and its optional response implemented by a single Web Service. It usually comprises many interactions, i.e. Web Services, between the same set of business partners. An inter-organizational business process cannot be described by a stateless protocol. The flow of interactions (Web Services) between the business partners depends on the business entity states that are a result of the interactions. Inasmuch the interactions must be executed in an agreed order. This flow is described by a choreography of Web Services.

Following the Web Services model, a business partner must not only register the Web Services for each of the interactions, but also the choreography of the Web Services in a registry. A potential business partner must search the registry for choreographies that are compliant to his own 'preferred' choreography. This means that a service requester must first search for business processes that seem to match according to their semantic description. For each of these business processes it must retrieve the choreography description. Next, it has to check that the retrieved local choreography is complementary to its own local choreography. Complementary means that the general flow of interactions specified in the choreography is identical, but the task in each interaction is the corresponding one, i.e. if one business partner invokes a service the other one must retrieve a service call. Furthermore, the requester has to check its support of each of the individual services within the choreography as specified by the service provider. It follows that searching for a complementary inter-organizational business process is not a simple query to a registry. It additionally requires matching with the own 'preferred' business process descriptions. Imagine that a SOA approach to inter-organizational systems is successful. This means a registry contains millions of business process descriptions. It becomes, evident that such a retrieval process does not scale.

\section{B. Bottom-up approach}

In the previous subsection we concluded that the retrieval process does not scale. Furthermore, we believe that it is even unlikely in a bottom-up approach to find complementary business processes in a registry. This is due to the fact that the choreographies - that are used to define a collaboration - are not developed in collaboration. Companies will use some kind of business process modeling method to describe the orchestration of their internal business processes. The local choreography is derived by a projection on this orchestration that includes only those activities that are visible to the outside world and require interaction with a business partner. If each business partner develops the local choreography in isolation from each other it is rather unlikely that the resulting business process models will match. Consequently, the business partners will not be able to perform an inter-organizational business process by electronic means.

It follows that the local choreographies of each partner should follow an agreed model of an inter-organizational business process - no matter whether it is agreed bi-laterally or it follows a standardized business process model. Such an agreed inter-organizational process serves more or less as a contract between the business partners with respect to their commitments and agreements on the business process level. This commitment and agreement model is described like any other contract from a neutral perspective leading to a so-called global choreography. Each business partner is than able to derive its local choreography and to bind its internal processes to this choreography. This approach increases the chance for finding complementary business processes. Furthermore, it leads to a retrieval process that will scale, since service requesters will search for business partners that support the same global choreographies in the complementary role - this may be easily realized by searching for a particular inter-organizational process ID. 


\section{Missing value perspective}

The development of an inter-organizational system does usually not consider the value proposition of participating in the inter-organizational process. It rather concentrates on the definition of the sequence and structure of the interorganizational business process. However, before two or more companies will engage in a collaborative process it is important to analyze how and why these enterprises cooperate from a business perspective. The answer to this questions is given by the value perspective as shown on top of figure 1. A business model - which differs from a business process model - has to define which value objects are created by which partner and which value objects are exchanged between which partners. This reveals the value proposition for each participating partner and indicates to the management whether participation in the inter-organizational business process is economically relevant or not. However, the value perspective is rarely used in traditional approaches or even left out completely.

\section{RESEARCH GOALS}

Aligning business with IT. The main aim of BSopt is to align service science engineering (in the context of management) with the respective services portfolio in a technical sense, resulting in $\mathrm{B} 2 \mathrm{~B}$ information systems according to the concept of a service-oriented architecture (SOA). A successful B2B integration does not solely concentrate on the technology layer. Accordingly, starting off at the technology layer by creating Web Services artifacts - e.g. WSDL - is not appropriate, as the business perspective, the justification for the business process and the business requirements capturing the commitments and agreements between business partners are not considered. In addition, if business partners design their own interfaces in isolation, it is rather unlikely that their interfaces are complimentary to each other. Instead, the business semantics must drive the technological implementations. Thus, it is important to consider the value perspective as well as business processes supporting this perspective when designing a service-oriented architecture for B2B. In other words, the business semantics must sit on top of the underlying process technology.

Consistent and integrated methodology for business services. This overall research goal is reached by providing a consistent and holistic methodology for business services. The management focuses on the value perspective in order to maximize a company's profit. In addition to revenue estimates, a resulting business model - on the top layer of figure 1 - captures the rationale as well as the economic resources being exchanged between business partners. A business model must be supported by a set of business process specifications. The process perspective manifested in the business process models - on the middle layer of figure 1 - specifies a flow of business activities and their dependencies specially designed to reach the business goals.

It is important to differentiate between two kinds of business process models: one kind describes the interorganizational choreography between business partners and another one describing the orchestration internal to a business partner. The first one serves as a kind of contract between business partners capturing the agreements and commitments on the exchanges between business partners. The latter one describes processes to produce and consume the resource/message exchanges - it is a black box to other business partners. Evidently, the two kinds of business process models must be in sync, since the internal business processes must interface with what was agreed in the interorganizational model. In summary, both kind of business process models describe "how" a business transaction is realized.

The business models and the resulting business processes of the two BOV layers of figure 1 must be supported by IT systems on the FSV layer. It is desired to configure a business service interface according to a process description e.g. a workflow engine is fed with a machine-readable representation of a business process. This machine-readable representation corresponds to the deployment artifacts on the third layer of figure 1 .

It is apparent, that each of the three layers of figure 1 uses different levels of abstractions to capture/implement what an enterprise does or wants to do. Accordingly each layer has its own specific methods resulting in an inconsistent, not harmonized view on the business itself. The goal of BSopt is to provide a consistent and integrated methodology for business services that spans over all layers of figure 1 .

Software Factory for BSopt. The BSopt methodology and its underlying meta model must be supported by a tool set. In the presented approach a domain specific language (DSL) will be used to support the specific needs of BSopt. The software industry has come up with meta frameworks for defining DSLs on top of it - a popular example are the Domain Specific Language Tools for Microsoft Visual Studio. In addition to these tools, business process tools like ADONIS provide meta modeling facilities, too. All these meta frameworks form the basis for the definition of a meta model, a foundation for a graphical modeling environment and frameworks to implement and automate code generation. The resulting environment is called a software factory, which corresponds to a modeling tool for a certain DSL plus user guidance by providing reusable patterns, receipts, guidelines, wizards, etc.

Integration of the methodology into software environments. As outlined in the paragraphs before, the BSopt methodology will deliver new ways of connecting business models, business process models and deployment artifacts, e.g. Web Service artifacts. This new working environment supported by an appropriate tool set must be integrated into a software environment that is already in place. Deployment artifacts generated with BSopt will be used in the real world IT-landscape of our project partner from the print media domain.

\section{RELATED WORK}

According to our three layered approach this section gives an overview about state-of-the-art approaches already existing on each layer. The top level layer describes how to formalize business models, which we consider as being a layer on top of business process models. There exist several methods to capture and model the economics behind the business process. The e3-Value ontology [3] is the most advanced approach which considers a business model as 
a value constellation - i.e., a network of enterprises that jointly creates and distributes objects of economic value. In contrast to the e3-value model, which describes the network constellation from a global point of view, the Business Model Ontology (BMO) [4] rather focuses on a specific actor and outlines his position in the business network and how he can make profit. As a third approach the ResourceEvent-Agent Ontology (REA) [5] captures the declarative semantics of the collaborative space between enterprises from an economic viewpoint. It describes the involved actors, their value exchanges and holds the triggers for economic exchanges by the means of economic events.

At the business process modeling layer a lot of approaches have been developed based on different notations. The Business Process Modeling Notation (BPMN) [6] standardized by the Object Management Group (OMG) incorporates aspects of already advanced modeling notations (e.g. UML activity diagrams, IDEF [7], ebXML BPSS [8], RosettaNet [9], etc.). Another very popular notation are the Event-driven Process Chains (EPC) focusing on control flow dependencies of activities in a business process. EPC are utilized in the ARchitecture of Integrated Information Systems (ARIS) [10]. In addition to special modeling notations UML can be customized for modeling business processes e.g. a UML Profile for Business Models [11]. In recent years a lot of research has been done by extending the UML meta model (e.g. UML activity diagrams). Most of these approaches focus on describing business processes internal to an organization fulfilling customer needs. In [12] a comparison of different approaches is given. Comparatively less work is spent on inter-organizational business process modeling. In BSopt we will use UN/CEFACT's Modeling Methodology (UMM) for modeling the global choreography of interorganizational business processes [11] [13]. UMM is a UML profile standardized by UN/CEFACT (United Nations Centre for Trade Facilitation and Electronic Business), known for its standardization work in the field of UN/EDIFACT and ebXML [14].

Another important artifact at the business process modeling layer are the business documents which are exchanged during an inter-organizational business process. Several publications already focused on this topic e.g. [15] [16]. The BSopt approach will employ the core components technology (CCTS) [17] as proposed by UN/CEFACT. Core components are reusable building blocks for assembling business documents. In order to allow a simple integration into a UML modeling tool of choice UN/CEFACT has also developed a UML profile for core components [18].

The third layer describes the deployment artifacts capturing the process specifications in order to make business process descriptions machine-interpretable. Process or workflow engines are fed with such business process specifications (mostly XML-based syntax) in order to adapt their behavior as required by the business process. In the BSopt approach we will use WS-BPEL [19] and XAML [20] as a description language for Windows Workflow. A survey of different XML-based business process languages is provided in [21]. Furthermore the integration approach of bringing these three layers together is an important issue. The most prominent example is generating code or process specifications follow- ing a model driven approach. The research work in [22] examines the BPMN-BPEL binding. In terms of UMM, work to map a global choreography defined in UMM to an ebXML business process specification [23] and to generate BPEL orchestrations from UMM business transactions [24] has already been done.

All these approaches of the three layers are considered by the BSopt project to develop a holistic methodology for inter-organizational systems. The need of holistic methodologies is not particularly new. Different frameworks have been developed to ensure a comprehensive approach to the planning, analysis, design, implementation, and governance of enterprise information systems. It is the special focus of BSopt on inter-organizational systems that it differs from approaches like the Zachman framework [25], The open Group Architecture Framework (TOGAF) [26], Sherwood Applied Business Security Architecture (SABSA) [27], the reference architectural styles for service-oriented computing as proposed in [28], as well as from various defense industry and government frameworks. Since all these frameworks are rather similar, we just highlight the concepts of the Zachman Framework which is the most commonly accepted one. It consists of a matrix, where the rows define the different participants' perspectives in building enterprise architecture (visionary, owner, designer, builder, implementer, and worker) and the columns represent the six basic interrogatives (what, how, where, who, when, and why). Each intersection contains a unique model giving an holistic view of the enterprise which is being modeled. Each model is technology neutral and does not identify a representation language. Therefore the Zachman approach was rather developed for analytical purposes than for developing concrete business process models. In [29] the authors propose to use a UML profile for the Zachman framework in order to overcome the shortcomings of having no well defined methodology and no concrete modeling notation.

Similar to our project, the European Union 6th Framework Project SUPER (Semantics Utilized for Process Management within and between Enterprises) [30] addresses the alignment of business process management and and existing IT systems and software. The major goal of this project is to raise business process management from the IT level to the business level. In contrary to the BSopt approach, SUPER does not explicitly provide any concepts to capture the value perspective of inter-organizational systems. Furthermore, SUPER and BSopt are based on different paradigms. SUPER is built upon technologies arising from the Semantic Web, in particular Semantic Web Services. In contrast BSopt uses a domain-specific language approach that explicitly defines the relevant business semantics in the underlying meta model.

\section{THE BSOPT APPROACH}

BSopt defines a methodology that considers business models, business process models, and deployment artifacts for a SOA. Business models may be expressed by the means of e3-Value, REA or BMO. We consider e3-Value the most promising approach, but complement it with some concepts defined in REA and BMO for capturing business models (step 1 in figure 2). On the business process layer we distinguish approaches to model the global choreography of 


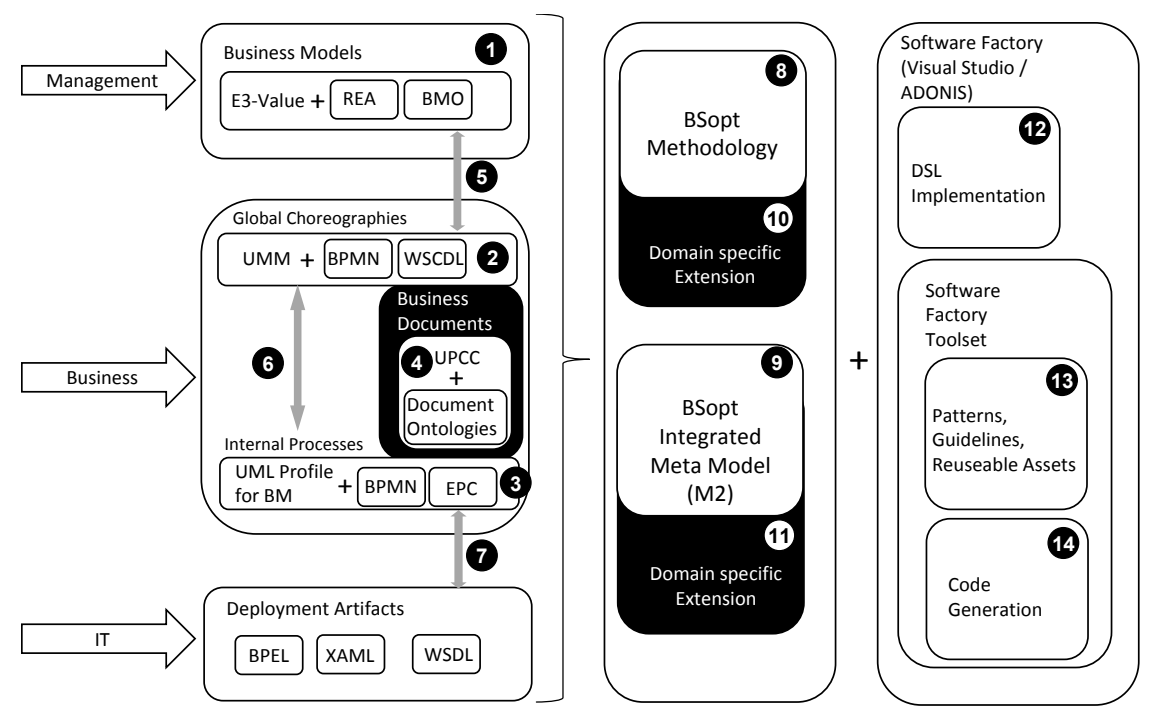

Fig. 2. The BSopt approach at a glance

an inter-organizational system and approaches to model the internal processes that provide an interface to the choreography. For global choreographies, we start off with UMM and will integrate concepts of BPMN and WS-CDL (step 2). For modeling internal processes we create a complement to the UMM consisting of concepts from the UML Profile for Business Modeling, UML activity diagrams, BPMN, and EPC (step 3). Business processes also involve business documents that are exchanged between business partners and are created/processed by the internal processes. Therefore, business documents must be considered both in global choreographies and in internal processes. In order to model business documents we base our approach on the UML Profile for Core Components and extend it in step 4 by approaches on document ontologies. On the deployment layer such an integration is not appropriate, because these standards have to stay as they are.

BSopt will integrate the approaches on each of the three layers. Thus, it is important to identify the dependencies between the approaches on each layer. This is a prerequisite to define a semi-automatic mapping between the artifacts on the different layers and to reflect changes on one layer to the other ones. Step 5 will investigate on the dependencies between business models and global choreographies, step 6 will elaborate on those between global choreographies and internal processes, and finally step 7 will define the mapping between internal processes including business documents and the various deployment artifacts.

Having defined the approaches on each layer and the dependencies between them, we will describe the overall BSopt methodology in step 8. The BSopt methodology will provide exact guidelines on the development process in order to create a BSopt compliant model spanning over all three layers. A model created by the BSopt methodology must be in accordance to the underlying BSopt meta model. The meta model must integrate all the semantics concepts on each layer and their interdependencies (step 9). This task is challenging because different languages are used to express the semantics of the approaches on different levels. Business model approaches are usually defined in prose without an explicitly defined meta model. Business process models may be specified in different languages, such as UML + certain profile extensions, EPC or BPMN. Deployment artifacts are usually XML based. Evidently, the conceptual BSopt meta model must be defined by a uniform language. In order to cope with the different requirements on each level we prefer a flexible knowledge representation language. Candidate languages for expressing the conceptual meta model are RDF Schema, OWL or MOF. In addition, the BSopt might be further tailored to a specific industry domain - as in our project to the domain of print media - in steps 10 and 11 .

As outlined in our research goals, the BSopt approach will not stay at a conceptual level, but will be implemented in a tool. For implementing BSopt, we follow the idea of a Software Factory [31]. Candidate platforms for the BSopt software factory are Microsofts DSL Tools for Visual Studio and ADONIS. In a first step the BSopt meta model defined in RDF Schema, OWL or MOF must be migrated to the meta language supported by the respective tool. This is subject to step 12, which has to consider both the BSopt methodology and the meta model. The implementation of the DSL concerns also the graphical representation of the modeling elements in the tool box and in the modeling canvas. The software factory relieves the user from redundantly repeated tasks at each level by providing semi-automatic generation of dependent artifacts. The implementation must also check the compliance of a model to the BSopt meta model. The compliance check applies consistency rules which cannot be expressed in the DSL of that tool. A key feature of a BSopt tool must be an intuitive user interface for the modeler. Whereas generic purpose modeling tools usually require a deep understanding of the modeling language, a specially customized software factory guides the modeler through the development processes in a wizard-driven style. Furthermore, patterns, guidelines and reusable assets will assist the modeler in creating BSopt compliant artifacts (step 13).

The ultimate goal of a software factory is generating code. In our case this corresponds to the generation of deployment artifacts (e.g., process orchestrations and workflow code) to 
be consumed by a software environment. According to step 14 in figure 2 we focus on generating BPEL and XAML based on the business semantics defined in the business models and in the business process models. Finally, the BSopt approach will be validated by conducting a real-world case study within the BSopt project together with the print media industry partner.

\section{CONCLUSION}

In this paper we introduced a top-down methodology called BSopt for designing inter-organizational systems backed by the concepts of SOA's. We discussed the shortcomings of the conventional bottom-up procedure when implementing a service-based system. We exhibited that the bottom-up approach starting from Web Services definitions does neither scale in the retrieval of business processes offered by potential business partners nor does it provide a reasonable chance to find complementary business processes. We further stressed that conventional requirements engineering employs business process modeling for eliciting process requirements, but ignores the value perspective of business processes. However, the value perspective - i.e., the view of the management - provides the stop or go for realizing an e-business scenario.

In order to overcome these limitations, we shaped our research goals for BSopt starting from the value perspective, leading over to business process design and resulting in IT deployment artifacts. Our research goals are based on the intention not to contrive yet another modeling approach, but to combine existing ones to an integrated top-down methodology. We discussed the steps in developing the BSopt methodology and which existing approaches are merged into BSopt. Furthermore, we stressed the fact that BSopt will be backed by a software factory. The software factory allows stakeholders to quickly create a business service interface supporting their respective part of the business collaboration. It becomes clear that our top-down approach is superior to existing bottom-up proceedings in realizing e-business based on the principles of SOA. Since we align management, business and IT perspectives in one methodology, a company implementing BSopt is flexible in adapting to changing market conditions.

\section{REFERENCES}

[1] Reference Model for Service Oriented Architecture, OASIS, 2006 Committee Specification 1.0.

[2] Open-edi Reference Model, ISO, 1997, ISO Standard 14662.

[3] J. Gordijn and H. Akkerman, "Designing and evaluating e-business models," IEEE Intelligent Systems, 2001.

[4] A. Osterwalder and Y. Pigneur, "An e-Business Model Ontology for Modeling e-Business," in Proceedings of the 15th Bled E-Commerce Conference - Constructing the eEconomy, 2002.

[5] G. Geerts and W. E. McCarthy, "The Ontological Foundation of REA Enterprise Information Systems," Michigan State University, Tech. Rep., 2000.

[6] BPMN Specification 1.0, OMG - Object Management Group, 2006.

[7] Integrated Definition Methods, IDEF. [Online]. Available: http://www.idef.org

[8] UN/CEFACT - ebXML Business Process Specification Schema, UN/CEFACT Techniques and Methodologies Group, Nov. 2003, Version 1.10. [Online]. Available: http://www.untmg.org/dmdocuments/BPSS_v110_2003_10_18.pdf

[9] RosettaNet Implementation Framework: Core Specification, RosettaNet, Dec. 2002, v02.00.01. [Online]. Available: http://www.rosettanet.org/rnif
[10] A.-W. Scheer, ARIS - Business Process Modeling. Springer, 2000.

[11] UN/CEFACT's Modeling Methodology (UMM), UMM Meta Model - Foundation Module, UN/CEFACT, Sep. 2006, Technical Specification V1.0. [Online]. Available: http://www.unece.org/cefact/umm/UMM_Foundation_Module.pdf

[12] B. Korherr and B. List, "An Evaluation of Conceptual Business Process Modelling Languages," in Symposium on Applied Computing (SAC'06). ACM Press, 2006.

[13] B. Hofreiter, C. Huemer, P. Liegl, R. Schuster, and M. Zapletal, "UN/CEFACT's Modeling Methodology (UMM): A UML Profile for B2B e-Commerce," in Advances in Conceptual Modeling - Theory and Practice, ER 2006 Workshops. Springer, 2006.

[14] ebXML - Technical Architecture Specification, OASIS, UN/CEFACT, February 2001, Version 1.4. [Online]. Available: http://www.ebXML.org/specs/ebTA.pdf

[15] D. Fensel, Y. Ding, B. Omelayenko, E. Schulten, G. Botquin, M. Brown, and A. Flett, "Product Data Integration in B2B ECommerce," IEEE Intelligent Systems and Their Applications, vol. 16, no. 4, pp. 54-59, 2001.

[16] J. Guo, "Inter-Enterprise Business Document Exchange," in Proceedings of the 8th international conference on Electronic commerce (ICEC06). ACM Press, 2006.

[17] Core Components Technical Specification - Part 8 of the ebXML Framework, UN/CEFACT Techniques and Methodologies Group, Nov. 2003, version 2.01. [Online]. Available: http://www.unece.org/cefact/ebxml/CCTS_V2-01_Final.pdf

[18] UML Profile for Core Components based on CCTS 2.01, UN/CEFACT Techniques and Methodologies Group, Feb. 2006, Candidate for Version 1.0.

[19] Web Services Business Process Execution Language, OASIS, 2007, V2.0.

[20] Extensible Application Markup Language, Microsoft Cooperation, 2007. [Online]. Available: http://msdn2.microsoft.com/enus/library/ms752059.aspx

[21] W. van der Aalst, A. Hofstede, and M. Weske, "Business Process Management: A Survey," 2003, BPM Center Report BPM-03-02.

[22] C. Ouyang, M. Dumas, A. Hofstede, and W. M. P. van der Aalst, "From BPMN Process Models to BPEL Web Services," in ICWS '06: Proceedings of the IEEE International Conference on Web Services (ICWS'06). IEEE Computer Society, 2006.

[23] B. Hofreiter, C. Huemer, and J.-H. Kim, "Choreography of ebXML business collaborations," Information Systems and e-Business Management (ISeB), 2006.

[24] B. Hofreiter, C. Huemer, P. Liegl, R. Schuster, and M. Zapletal, "Deriving executable BPEL from UMM Business Transactions," in Proceedings of the IEEE International Conference on Services Computing (SCC07). IEEE Computer Society, Jul. 2007.

[25] J. Zachman, "A Framework for Information Systems Architecture," IBM Systems Journal, vol. 31, no. 3, pp. 445-470, 1999.

[26] The Open Group, The Open Group Architecture Framework, 2006, tOGAF 8.1.1.

[27] J. Sherwood, A. Clark, and D. Lynas, Enterprise Security Architecture: A Business-Driven Approach. cmp, 2005.

[28] T. S. Dillon, C. Wu, and E. Chang, "Reference Architectural Styles for Service-Oriented Computing," in Network and Parallel Computing, IFIP International Conference (NPC 2007), vol. 4672. Springer LNCS, 2007, Keynote.

[29] Fatolahi, Ali, Shams, and Fereidoon, "An investigation into applying UML to the Zachman framework," Information Systems Frontiers, vol. 8, no. 2, pp. 133-143, February 2006.

[30] "IP Super - Integrated Project for Semantics Utilized for Process Management within and between Enterprises," European Union 6th Framework - Information and Society Technologies (IST). [Online]. Available: http://www.ip-super.org

[31] J. Greenfield, K. Short, S. Cook, S. Kent, and J. Crupi, Software Factories: Assembling Applications with Patterns, Models, Frameworks, and Tools. Wiley, 2004. 